\title{
MOBILE SENSOR-ACTUATOR NETWORKS: OPPORTUNITIES AND CHALLENGES
}

\author{
MARTIN HAENGGI \\ Deptartment of Electrical Engineering, University of Notre Dame \\ Notre Dame, IN 46556, USA \\ haenggi@ieee.org
}

\begin{abstract}
Large-scale networks of integrated wireless sensors and actuators become increasingly tractable. Advances in hardware technology and engineering design have led to dramatic reductions in size, power consumption, and cost for digital circuitry, wireless communications, and MEMS. This enables very compact, autonomous, and mobile nodes, each containing one or more sensors and actuators, computation and communication capabilities, and a power supply. Networking is a crucial ingredient to harness these capabilities into a complete system. While wireless sensor networks have been studied for about a decade, their extension with actuators is a more recent thrust of research that greatly enhances their capabilities and range of applications, at the cost of requiring closed control loops that can cause instability and are subject to delay constraints. This article provides an overview over existing and emerging technologies, pointing out the opportunities and challenges of mobile integrated sensor-actuator networks and their relation to CNNs.
\end{abstract}

\section{Introduction}

Pervasive micro-sensing and -actuation may revolutionize the way we understand and manage complex physical systems. The capabilities for detailed physical monitoring and manipulation offer enormous opportunities for almost every scientific discipline. Networks of such devices, so-called sensor-actuators networks (SANs) provide an embedded processing platform with exciting capabilities ${ }^{1,2}$.

Applications include surveillance in inhospitable environments such as remote geographic regions or toxic locations, sensing and maintenance in large industrical plants, planetary exploration, seismic activity detection, medical sensing, micro-surgery, military surveillance and combat, fingertip accelerometer virtual keyboards, and smart office spaces.

In practically all such applications, key requirements include scalability, robustness with respect to various disturbances and uncertainties, evolvability through autonomous reconfiguration and optimal redistribution of resources, and interoperability in heterogeneous system environments ${ }^{3}$. In addition, verifiability of the underlying properties and features of the resulting overall distributed network must be achievable at various levels of computational complexity.

Wireless connectivity is crucial, since for most envisioned applications, the environment being monitored does not have installed infrastructure for either communications or energy. Therefore untethered nodes must relay on small local energy sources, as well as wireless communication channels. Due to energy and interference considerations, the transmission range of a node will only include its nearest neighbors, and since computation is substantially cheaper than transmission, control and resource allocation algorithms run locally on every node in a distributed fashion. With these properties, an SAN can be clearly viewed at as a CNN. 
From a networking point of view, SANs belong to the class of mobile ad hoc networks (MANET) ${ }^{4,5}$. Section 3 includes a characterization of MANETs and discusses the resulting networking challenges for the design of SANs.

\section{Implementation of Network Nodes}

In this section, we will give an overview over a few of the existing and emerging technologies; a more complete list and details on protocols and their implementations can be found in ${ }^{5}$. Since the prerequisite of any implementation is a power supply with sufficient performance, we will discuss the power and energy issue first.

\subsection{Power Supply}

The most difficult constraints in the design of SANs are those regarding the minimum energy consumption necessary to drive the circuits and MEMS devices. The energy problem is aggravated by the actuators that may be substantially more power-hungry than the sensors. When miniaturizing the node the energy density of the power supply is the primary issue. Current technology yields batteries with approx. $1 \mathrm{~J} / \mathrm{mm}^{3}$ of energy, while capacitors can achieve as much as $1 \mathrm{~mJ} / \mathrm{mm}^{3}$.

Given only the option of a battery for a power source, the functionality of a node would be limited. If a node were designed to have a relatively short lifespan, such as a device built to transmit data only a few times, a battery would be a logical solution. However, for nodes that can generate sensor readings for long periods of time, a charging method for the supply must be utilized. Currently, research groups investigate the use of solar cells to charge capacitors with photocurrents from the ambient light sources. Solar flux can yield power densities of approximately $1 \mathrm{~mW} / \mathrm{mm}^{2}$. The energy efficiency of a solar cell ranges from $10-30 \%$ in current technologies, giving $300 \mu \mathrm{W}$ in full sunlight in the best-case scenario for a $1 \mathrm{~mm}^{2}$ solar cell operating at 1V. Series-stacked solar cells will need to be utilized in order to provide appropriate voltages.

Sensor acquisition can be achieved at $1 \mathrm{~nJ} /$ sample, and modern processors can perform computations as low as $1 \mathrm{~nJ} /$ instruction. For wireless communications, the primary candidate technologies are based on RF and optical transmission techniques. Each technique has its advantages and disadvantages. RF presents a problem because the nodes may offer very limited space for for antennas, thereby demanding very short-wavelength (i.e., high-frequency) transmission. Communication in that regime is not currently compatible with low power operation. Current RF transmission techniques (e.g., Bluetooth ${ }^{6}$ ) consum about 100nJ/bit for a distance of $10-100 \mathrm{~m}$, making communication very expensive compared to acquisition and processing. An attractive alternative is to employ free-space optical transmission. If a line-of-sight path is available, a well-designed free-space optical link requires significantly lower energy than its RF counterpart, currently about $1 \mathrm{~nJ} /$ bit. The reasons for this power advantage are that optical transceivers require only simple baseband analog and digital circuitry and no modulators, active filters, and demodulators. The extremely short wavelength of visible light makes it possible for a millimeter-scale device to emit a narrow beam, corresponding to an antenna gain of roughly 7 orders of magnitude compared to an isotropic radiator.

7th IEEE International Workshop on CNNs and their Applications 
In SANs, where sensor sampling, processing, data transmission, and actuating is involved, the trade-off between these tasks play an important role in power usage. Given a finite amount of energy, balancing these parameters depending on the application will be the focus of the design process of SANs.

\subsection{An Example: Berkeley "Smart Dust"}

The Smart Dust project at the University of California at Berkeley $7,8,9$ aims at exploring the limits of system miniaturization by packing an autonomous sensing, computing, and communication node into a so-called "mote" with a volume of one cubic millimeter. This ambitious project requires both evolutionary and revolutionary advances in miniaturization, integration, and energy management. Such advances will be facilitated by the progress in MEMS, which permits the fabrication of small sensors, optical communication components, actuators, and power supplies. Microelectronics provides the necessary functionality and processing power in ever smaller areas with decreasing power and energy consumption. The Berkeley group follows two approaches, one is based on RF communication, the other on optical links.

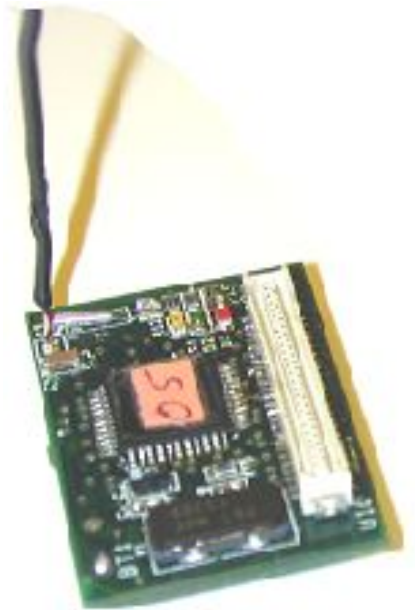

Figure 1. The "Berkeley mote" RF wireless node..

RF motes. The current state of the RF mote (Fig. 1) is a matchbox sized PCB with off-the-shelf components: A $4 \mathrm{MHz} 8$ bit CPU (by Atmel), a 50kbit/s transceiver in the $900 \mathrm{MHz}$ band (by RF Monolithics), $4 \mathrm{~KB}$ data memory, $128 \mathrm{~KB}$ program memory, and $6 \mathrm{~A} / \mathrm{D}$ channels to convert the sensor data. Various types of sensors such as accelerometers and magnetic, temperature, light, humidity, acoustic and pressure sensors can be connected via a generic interface. An extremely compact operating system called "TinyOS" has been tailor-made for this type of mote $^{10}$.

Of course this mote is far from fitting in a cubic millimeter, but merely by combining its components into a single chip, its volume could be decreased by two orders of magnitude.

Optical motes. As discussed in the Section on power supply, a promising alternative to RF communication are laser-based optical links. The main disadvantage is that a line-of-sight path has to be available, and the main challenge is to aim the laser beam precisely at the receiving mote. Additionally, the laser diodes are relatively power hungry. The Berkeley group managed to come up with an elegant solutions to these problems by implementing a passive transmission technique ${ }^{7}$, i.e., to modulate incoming optical signals and reflect them. This idea is based on a corner-cube retroreflector (CCR) that comprises three mutually perpendicular 


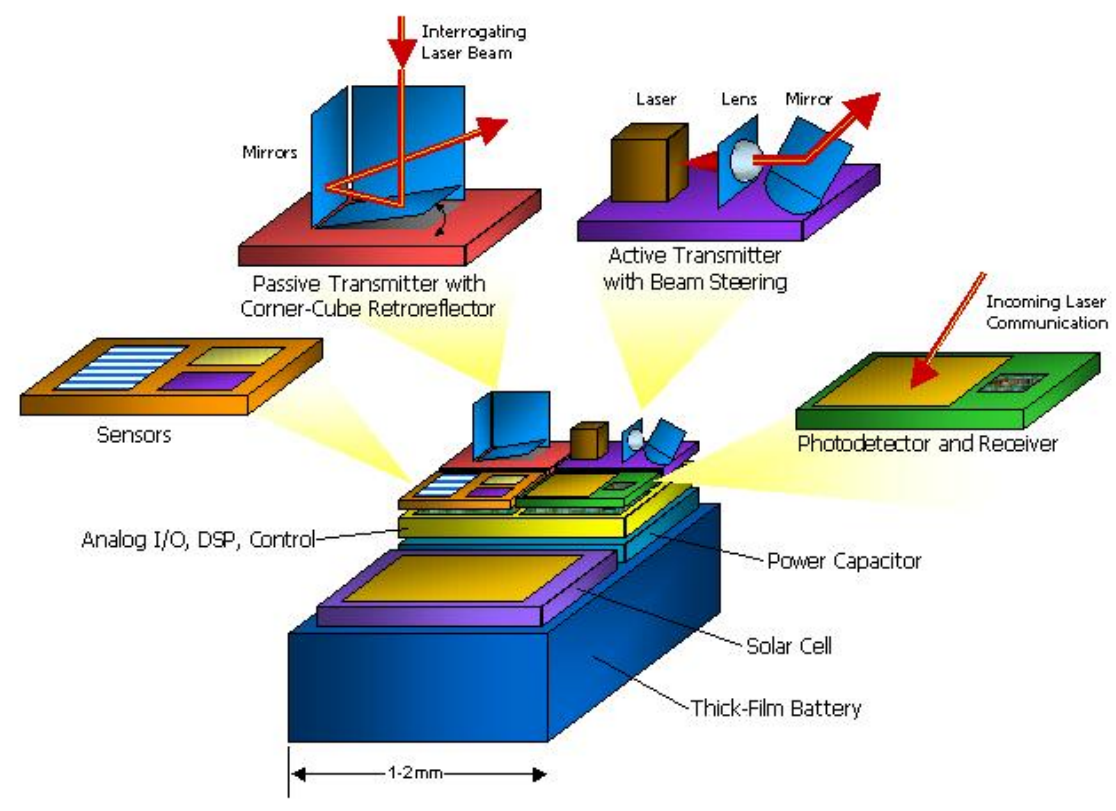

Figure 2. Optical Berkeley mote. (C) UC Berkeley.)

mirrors of gold-coated polysilicon. The CCR has the property that any incident ray of light is reflected back to its source. The MEMS based CCR includes an electrostatic actuator that can deflect one of the mirros at kilohertz rates. It has been demonstrated that a CCR illuminated by an external light source can transmit back a modulated signal at kilobits per second. Figure 2 presents an optical mote with both passive and active communication. For the active transmitter, MEMS technology can be used to assemble a beam-steering micro-mirror to enable communication between nodes. For passive transmission, it is assumed that the operator of the SAN is using a relatively high power laser beam to "interrogate" the motes (Fig. 3). Such an interrogating beam could be built in binoculars, for example. For the receiver, a CCD camera is suggested, that will have to detect numerous incoming signals simultaneously (Fig. 4). For an envisioned size of up to $256 \times 256$ pixels, this CCD array will generate a data flow of several tens of Mbits/s, which cannot be handled in a hand-held device. Clearly, the incoming data carried by the modulated reflected laser beams has to pre-processed and/or compressed early, most beneficially directly at the CCD sensor. Such a task is certainly tailored to CNNs with optical inputs, since the CNN processing capability permits an efficient detection of relevant patterns in the motes' response.

\subsection{Combining Sensors and Actuators}

The Berkeley platform includes mirrors as actuators in the optical mote, but that does not enable it to react to the environment. Numerous applications only become attractive if the nodes are mobile and are able to react to what they see or what

7th IEEE International Workshop on CNNs and their Applications 


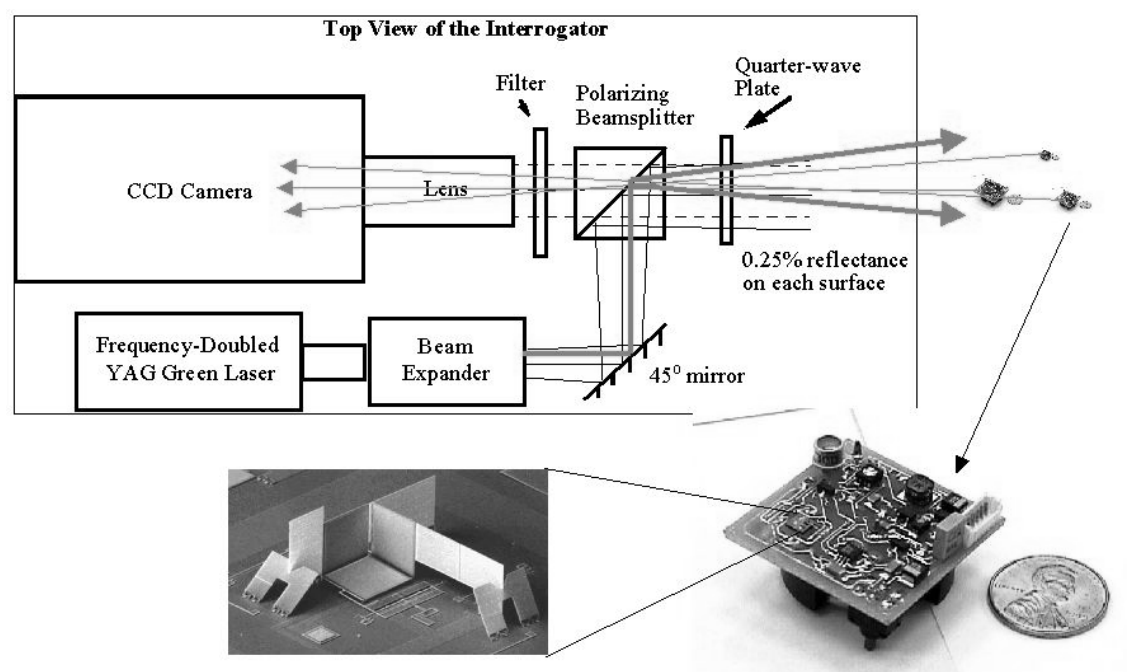

Figure 3. Interrogator scheme for the optical network node. (C) UC Berkeley.)

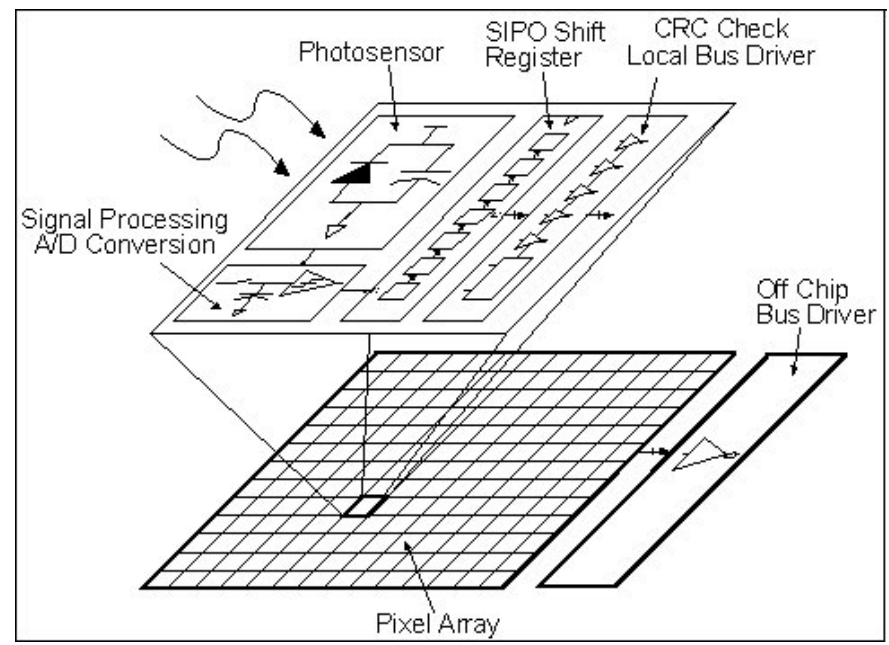

Figure 4. Receiver structure of the optical Berkeley mote. Replaced by a CNN with optical input, the receiver can interpret incoming data. (C) UC Berkeley.)

the network as a whole sees. Mobile platforms could include small mobile robots or flying insect-like nodes. Such an extension necessarily entails closed sensor-actuator control loops. Since sensor and actuator are generally not colocated on one node, the control problem is distributed, and it suffers from unknown delays induced by the underlying network. Closed-loop control aspects of large networks are the 
subject of a research effort sponsored by DARPA/OIT ${ }^{a}$. This work is currently in progress; the goal is the formal verification of the correctness of the distributed control algorithm, and to derive tight upper bounds on the delay of the feedback loops. As with CNNs, such feedback leads to much richer dynamics but necessitates measures to guarantee stability. Some of the challenges are caused by the properties of the communication network, which are discussed in the next Section.

\section{Networking Issues}

\subsection{SANs as mobile ad hoc networks}

As mentioned earlier, SANs are mobile ad hoc networks (MANETs) ${ }^{4,5}$. MANETs have several salient characteristics:

- Dynamic topologies: the network topology may change randomly and rapidly at unpredictable times. A connection between two nodes usually uses several intermediate nodes as relays (multi-hop connection).

- Bandwidth-constrained, variable capacity wireless links

- Energy-constrained operation

- Limited physical security

These properties pose formidable challenges to SAN implementations, one of which is the routing problem. How can a path be found from one node to a possibly distant destination node if the topology is dynamic, individual nodes are not reliable and only nearest neighbors can be reached directly?

\subsection{Routing and clustering}

Numerous routing algorithms have been proposed and studied for ad hoc networks ${ }^{11}$, but most of them suffer from the drawbacks that they require globally unique addresses and that entire routes from end-node to end-node have to be discovered and continuously updated. The so-called directed diffusion ${ }^{12}$ algorithm takes a different approach by letting messages pass from node to node, without specifying an exact destination node but rather a geographic area or even just a request for certain information. Nodes only have to be aware of their nearest neighbors; this guarantees that a node that has the desired information can reply via the reverse path. Such a diffusion mechanism is quite similar to the diffusion process in CNNs, which was rigorously analyzed in ${ }^{13}$.

Nearest-neighbor awareness naturally leads to a clustered network. The division of large networks into clusters consisting of cooperating nodes has several advantages such as increased robustness and security; simplified addressing, routing, and localization; lower energy consumption, and lower memory requirements ${ }^{14,15}$. Furthermore, since sensors and actuators can be assumed to be in the same cluster, the delay in the control loop will be reduced. Such a clustered SAN can also be referred to as cellular SAN, in accordance with cellular wireless networks used in mobile telephony.

${ }^{a}$ Office of Information Technology at the Defense Advanced Research Project Agency, USA 


\section{Conclusions and Outlook}

SANs are a very promising platform for pervasive sensing, surveillance, and computing which may revolutionize information gathering and processing. As miniaturization proceeds further, goals such as cubic millimeter "smart dust" become feasible. As Kris Pister, one of the Smart Dust investigators, points out: "In 2010 MEMS sensors will be everywhere, and sensing virtually everything. Scavenging power from sunlight, vibration, thermal gradients, and background RF, sensors motes will be immortal, completely self contained, single chip computers with sensing, communication, and power supply built in. Entirely solid state, and with no natural decay processes, they may well survive the human race."

The discussion of SANs revealed that they are related to CNNs in multiple ways. Not only the topology with its nearest-neighbor connectivity is similar, but also the process how information is diffused or propagated through the network. Furthermore, CNNs can be readily applied as receivers for networks with optical communication, and stability studies of SAN control loops may benefit considerably from results in CNNs theory. Another promising application in the realm of SANs is the use of CNNs for multisensor fusion ${ }^{16}$.

Networking with such tiny nodes is closely related to the concept of nanonetworking recently introduced by the author, which denotes networking with nodes that are based on nanoelectronic devices. CNNs based on resonant tunneling $\operatorname{diodes}^{17}$ or quantum dots ${ }^{18,19,20}$ are the first examples of such nanonets. In the context of this article, wireless nanonets are envisioned that have the potential to surpass the barrier of $1 \mathrm{~mm}^{3}$ per node, thus coming even closer to real smart dust.

\section{Acknowledgment}

The partial support of the DARPA/ITO-NEST Program (AF-F30602-01-2-0526) is gratefully acknowledged.

\section{References}

1. Gregory J. Pottie and W. J. Kaiser. Wireless integrated network sensors. Communications of the ACM, 43(5):551-558, 2000.

2. D. Estrin, L. Girod, G. Pottie, and M. Srivastava. Instrumenting the world with wireless sensor networks. In International Conference on Acoustics, Speech 83 Signal Processing, volume 4, pages 2033-2036, Salt Lake City, UT, 2001.

3. D.L. Hall and J. Llinas (editors). Handbook of Multisensor Data Fusion. CRC Press, 2001.

4. Internet Engineering Task Force. Mobile Ad-hoc Networks (MANET). http://www.ietf.org/html.charters/manet-charter.html.

5. C-K. Toh. Ad Hoc Mobile Wireless Networks - Protocols and Systems. Prentice-Hall, 2002. ISBN 0-13-007817-4.

6. Bluetooth Wireless Technology. Official Bluetooth site: http://www.bluetooth.com.

7. Joseph M. Kahn, Randy Howard Katz, and Kristofer S.J. Pister. Emerging 
challenges: Mobile networking for 'smart dust'. Journal of Communications and Networks, 2(3), September 2000.

8. B. Atwood, B. Warneke, and K.S.J. Pister. Preliminary Circuits for Smart Dust. In Southwest Symposium on Mixed-Signal Design, pages 87-92, San Diego, CA, February 2000.

9. B. Warneke, B. Atwood, and K.S.J. Pister. Smart dust mote forerunners. In 14th IEEE International Conference on MEMS, pages 357-360, 2001.

10. J. Hill, R. Szewczyk, A. Woo, D. Culler, S. Hollar, and K. Pister. System Architecture Directions for Networked Sensors. In International Conference on Architectural Support for Programming Languages and Operating Systems (ASPLOS), Cambridge, MA, November 2000.

11. Charles E. Perkins, editor. Ad Hoc Networking. Addison Wesley, 2000. ISBN 0-201-30976-9.

12. Chalermak Intanagowiwat, Ramesh Govindan, and Deborah Estrin. Directed diffusion: A scalable and robust communication paradigm for sensor networks. In Sixth Annual International Conference on Mobile Computing and Networking (MobiCOM), pages 56-67, Boston, MA, August 2000.

13. Patrick Thiran, Gianluca Setti, and Martin Hasler. An Approach to Information Propagation in 1-D Cellular Neural Networks - Part I: Local Diffusion. IEEE Transactions on Circuits and Systems-I, 45(8):777-789, August 1998.

14. I. Chlamtac and A. Farago. A new approach to the design and analysis of peer-to-peer mobile networks. Wireless Networks, 5:149-156, 1999.

15. A. Bruce McDonald and Taieb F. Znati. Design and Performance of a Distributed Dynamic Clustering Algorithm for Ad-Hoc Networks. In Simulation Symposium, pages 27-34, August 2001.

16. P. Arena, S. Baglio, L. Fortuna, and S. Graziani. Analog Cellular Networks for Multisensor Fusion and Control. IEEE Transactions on Circuits and SystemsI, 47(9):1378-1382, September 2000.

17. Martin Hänggi and Leon O. Chua. Cellular Neural Networks Based on Resonant Tunneling Diodes. International Journal of Circuit Theory and Applications, 29(5):487-504, Sep./Oct. 2001.

18. G. Toth, C.S. Lent, P.D. Tougaw, Y. Brazhnik, W. Weng, W. Porod, R.-W. Liu, and Y.-F. Huang. Quantum cellular neural networks. Superlattices and Microstructures, 20(4), 1996.

19. W. Porod. Towards Nanoelectronics: Possible CNN Implementations using Nanoelectronic Devices. In IEEE International Workshop on Cellular Neural Networks and their Applications, pages 20-25, London, 1998.

20. C. Gerousis, S. M. Goodnick, and W. Porod. Toward nanoelectronic cellular neural networks. International Journal of Circuit Theory and Applications, 28(6):523-535, Nov/Dec. 2000. Special issue on nanoelectronic circuits. 